\title{
Referee 65.
}

There is no discussion about the quality of the different extrapolation schemes considered in the manuscript (eqs. 1-5). This point should be discussed in the text and thus contribute to elucidate whether a given extrapolation scheme is more adequate than the others or not. At least, this will be valuable to provide some guidelines to discriminate between the energy differences provided by the various extrapolation schemes

We did not provide reader by the discussion of the quality of the different extrapolation schemes because in our opinion it is impossible. Our argument is following. Each extrapolation scheme, when it was introduced, produces perfect correspondence between reference data and the result of extrapolation. However, one never knows how good the our results for the subject of new study. Formally all schemes should converge to the

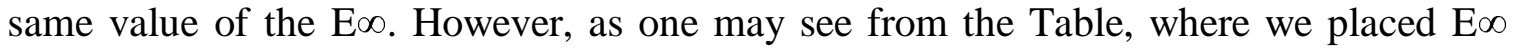
calculated for the schemes considered in our paper, this is not the case.

\begin{tabular}{|c|c|c|c|c|c|}
\hline & $\begin{array}{l}\mathrm{E}=\mathrm{E} \infty+\mathrm{Aexp}(- \\
\mathrm{BX}) \mathrm{X=2,3,4}\end{array}$ & $\begin{array}{l}\mathrm{E}=\mathrm{E} \propto 0+\mathrm{A} / \mathrm{X}^{3}+\mathrm{B} / \mathrm{X}^{4} \\
\mathrm{X}=\mathbf{2 , 3 , 4}\end{array}$ & $\begin{array}{l}\mathrm{E}=\mathrm{E} \propto+\mathrm{A} / \mathrm{X}^{3}+\mathrm{B} / \mathrm{X}^{5} \\
\mathrm{X}=\mathbf{2 , 3 , 4}\end{array}$ & $\begin{array}{l}\mathrm{E}=\mathrm{E} \propto+\mathrm{A} / \mathrm{X}^{4}+\mathrm{B} / \mathrm{X}^{5} \\
\mathrm{X}=\mathbf{2 , 3 , 4}\end{array}$ & $\begin{array}{l}\mathrm{E}=\mathrm{E} \propto 0+\mathrm{A} / \mathrm{\lambda} \\
\mathrm{X}=\mathbf{2 , 3}\end{array}$ \\
\hline $\begin{array}{l}\text { 9H-oxo- } \\
\text { amino } \\
\text { (a.u) }\end{array}$ & -541.842850 & -541.811190 & -541.780760 & -541.864240 & -541.81565 \\
\hline
\end{tabular}

Therefore, instead of discussing the quality of the available schemes we simply have chosen several the most popular extrapolation schemes.

The following paragraph has been added in the text:

We did not attempt to establish which of the extrapolation methods gives the most reliable CBS limits. For such a purpose one would need the results for $X=5$ which can not be obtained with available computational resources. Only a little is known about differences between CBS extrapolation schemes for large molecules of the size of guanine. Most of the validation of these formula comes from the study of small systems and shows only a little distinction between most of them. ${ }^{17-23,27}$ Hence, only the plain CBS limits for relative energies will be presented.

There is no comparison of present results with those reported previously in the literature (refs. 11-13). Indeed, more recent studies should also be mentioned. I quote, for instance, the study by Hanus et al. (JACS 2003, 125, 7678). Again, such a comparison should be useful to discuss the merits of the different extrapolation schemes

The following paragraph has been added.

A related study ${ }^{10 d}$ of the guanine tautomers, inlcuding the effect of their hydration, has been recently published by Hanus et al. The results of these investigations will be compared with our data. 
c) An interesting strategy (not used here) to estimate more reliably the energy differences between tautomers/rotamers in Table 1 would be to combine the values extrapolated from MP2 calculations with the higher-order correction estimated from CCSD(T) and MP2 calculations with the aug-cc-pvdz basis. Looking at the results in Table 1, it is clear that this latter correction destabilizes by $0.6 \mathrm{kcal} / \mathrm{mol}$ the 9H-syn-hydroxoamino structure, a feature that would increase the energy difference between $7 \mathrm{H}$-oxoamino and $9 \mathrm{H}$-syn-hydroxoamino as determined from eq. $5(0.28 \mathrm{kcal} / \mathrm{mol})$, and thus change the population of the chemical species given in Tables 2 and 3.

We believe that the most important is the comparison of our relative Gibbs free energies with the ones obtained by Hanus et al. (JACS 2003, 125, 7678). These data predict the same tautomers to be observable in equilibrium mixture and they are in close correspondence with those our results that predict predominance of $7 \mathrm{H}$-oxo-aminoguanine tautomer. Saying more specifically the results obtained by Hanus et al. (see Table3) are just slightly differ comparing with our CCSD(T)/aug-ccpvdz//MP2/augccpvdz data only for 9H-syn-hydroxo-amino-guanine tautomer and are virtually the same for all other cases

Taking this statement into account the revised paragraph looks as following:

Most of the computed data, including those recently published by Hanus et al. ${ }^{10 d}$, suggest that 7H-oxo-amino-tautomer should be the dominant form of guanine. Only in two cases which correspond to MP2/6-31G(d,p) and MP2/6-311++G(d,p) data the $9 \mathrm{H}$ oxo-amino form is dominant. It is also interesting to note that results of MP2/6$311++G(d, p), M P 2 / a u g-c c-p v d z$ calculations and the present CBS estimates predict almost the same fraction of the $9 \mathrm{H}$-oxo-amino- and $9 \mathrm{H}$-syn-hydroxo-amino-tautomers as that found by Hanus et al. ${ }^{10 d}$

In relation to the preceding point, I strongly disagree from the rationale presented in page 10 to determine the kinetics of the conversion from $9 \mathrm{H}-0$ xo to $9 \mathrm{H}$-hydroxo forms. In the forth paragraph, the authors mention the different populations of those tautomers obtained depending on the level of theory. Thus, results predicted from both MP2/6-31G(d,p) and CCSD(T)//aug-cc-pvdz calculations predict around 90\% population for the $9 \mathrm{H}$-oxo form. In contrast, MP2 calculations with 6$311++G(d, p)$ and aug-cc-pvdz basis predict similar populations for the two tautomers. Then, to discriminate between the two options they examine the available experimental data. However, what is unacceptable is to exploit crystallographic data nor data from physiological (aqueous) environments to determine the correct population in the gas phase. So, their choice for the population ratio determined from MP2/6-311++G(d,p) computations is in my opinion arbitrary and not well justified. 
First of all we would like to highlight some misunderstanding between us and Referee65. We did not explore crystallographic data nor data from physiological (aqueous) environments to determine the correct population in the gas phase. We made the point that the source of 'rare' tautomers for IR gas phase experiments is evaporation of guanine molecules from crystal which does not have observable concentrations of hydroxoforms. The same applies for the situation in living cell, since we do not know the biological mechanisms which result in synthesis of 'rare' tautomers of DNA bases. Therefore, suitable kinetics is the decay of $9 \mathrm{H}$-oxo-amino-guanine form. To clarify this point the corresponding paragraph has been revised as follows.

With the 7H-oxo-amino- tautomer excluded from further considerations the composition of thermodynamically equilibrated mixture will be reduced to one of the following two possibilities. According to the results of the $M P 2 / 6-31 G(d, p)$ and CCSDT/aug/cc-pvdz//MP2/aug-cc-pvdz approximations the mixture will be dominated by the $9 H$-oxo-amino-guanine form (more than 90 per cent). Alternatively, as suggested by the MP2/6-311++G(d,p) and MP2/aug-cc-pvdz data and by the results published in ref $10 \mathrm{c}$ the mixture should contain approximately equal concentrations of 9H-oxoamino- and 9H-syn-hydroxo-amino-forms. The latter results predict approximately the same concentrations of 9H-oxo-amino- and $9 H$-syn-hydroxo-amino-guanine species. To be more specific let us focus on the MP2/6-311++G(d,p) data. Since the $9 H$-oxo-aminoguanine is dominant in both crystals and living cells, the acceptable kinetics of proton transfer may anticipate the 9H-oxo-amino-form as the reagent and different hydroxoforms as products. Following these considerations, the results of $M P 2 / 6-311++G(d, p)$ calculations predict at $298.18 \mathrm{~K}$ the ratio of $9 H$-oxo-amino : $9 H$-syn-hydroxo-amino : 9H-anti-hydro-amino-forms as given by $51.5: 48.1: 0.4$.

\section{Again, I think that the best approach to solve the problem presented by the authors would be to use a composite strategy (see before; point c) to derive the best estimates for the energy differences between tautomers. Otherwise, the authors should also discuss the kinectics of the conversion between $9 \mathrm{H}-0 x \mathrm{x}$ and $9 \mathrm{H}$-hydroxo tautomers in the other scenario presented in page 10.}

In our opinion there is still no guarantee that our data (see the Table below) nor the data of Hanus et al. are converged with the accuracy let's say $0.2 \mathrm{kcal} / \mathrm{mol}$. Therefore, we adopt the second suggestion of referee to consider other cases. The following paragraph has been added.

However, the influence of the mentioned factors should also be investigated with care, since, for example, recent data suggest that the hydration by a selected number of water molecules can either stabilize or destabilize canonical forms of guanine, depending on the position of hydration. ${ }^{39}$

Finally, we would like to analyze briefly the chemical and biological consequences which follow from the data which predict the predominance of the $9 H$-oxoamino-guanine form. This particular case can be considered in terms of the MP2/6- 
$31 G(d, p)$ and $C C S D(T)$-aug-cc-pvdz//MP2/aug-cc-pvdz results. The former data are of low quality concerning both the basis set and the level of approximation. Therefore, we shall consider only CCSD(T)/aug-cc-pvdz//MP2/aug-cc-pvdz results and use the rate constants obtained at the MP2/6-311++G(d,p) level.

According to the CCSD(T)/aug-cc-pvdz//MP2/aug-cc-pvdz data the ratio of $9 H$ oxo-amino : 9H-syn-hydroxo-amino : $9 H$-anti-hydro-amino- forms is $96.1: 1.1: 2.8$. Hence, in this case the time to reach equilibrium should be quite short. Its present estimate amounts to about 50 hours. Since this time still is much longer then $1000 \mathrm{sec} \approx$ 0.3 hours, we conclude that the biological implications do not depend on the level of theory applied in the study.

There are several concepts that should be clearly stated. For example, a clear distinction between tautomers (9H-oxoamino; 9H-hydroxoamino) and conformers (9H-syn-hydroxoamino; 9H-anti-hydroxoamino) should be made and maintained along the text (see for instance paragraph 3 in page 5 , paragrapgh 2 in page 6 , and paragraph 5 in page 8).

The revised sentence of the $3^{\text {rd }}$ paragraph on page 5 reads as follows:

However, the conclusion concerning the local flatness of the potential energy surface has been later reconsidered and revoked in the investigations of four guanine species $^{6}$ (here they are 9H-oxo-amino-, 9H-syn-hydroxo-amino-, 7H-oxo-amino-, 9Hanti-hydroxo-amino-guanine), and two transition states for the oxo-hydroxo proton transfer (see the Scheme 1 above).

The revised sentences of the $2^{\text {nd }}$ paragraph on page 6 reads as follows:

However, because of steric interactions this form should be less favoured. Indeed, according to our pilot calculations the $7 \mathrm{H}$-anti-hydroxo-amino-guanine has been found to lie by more than $10 \mathrm{kcal} / \mathrm{mol}$ higher than the lowest-energy tautomer of guanine and thus this tautomer was not included in more accurate studies carried out afterwards. In addition to the remaining five species we have investigated the transition states for the proton transfer between the oxo- and hydroxo-forms.

The revised sentence of the $5^{\text {th }}$ paragraph on page 8 reads as follows:

One finds that the four lowest energy species all lie within the energy range of less than $2 \mathrm{kcal} / \mathrm{mol}$

Moreover, the terms syn and anti should be defined in the text.

To define tems 'syn-' and 'anti-' we have placed unfigure notes on Scheme I.

Finally, where "the conclusion concerning the local flatness of the potential energy surface has been later reconsidered" (paragraph 3 in page 5)?. No reference is given to support this latter statement. 
The reference has been added.

Page 6, line 2. Ref 13 is not a work reported by the authors. So, the sentence must be changed or the reference " 13 " be eliminated.

Reference 13 has been eliminated from the mentioned sentence.

* The tautomers/conformers examined in this study must be explicitly denoted in page 6 (paragragh 2). What are the "more accurate studies carried out afterwards"? Again, some references are required here.

The revised sentences read as follows:

These six structures are 9H-oxo-amino-guanine, 9H-syn-hydroxo-amino-guanine, 9Hsyn-hydroxo-amino-guanine, 9H-anti-hydroxo-amino-guanine, $7 H$-syn-hydroxo-aminoguanine, $7 \mathrm{H}$-anti-hydroxo-amino-guanine. They include the $7 \mathrm{H}$-anti-hydroxo-aminotautomer which has not been investigated earlier. ${ }^{11-13}$

I assume that the nature of all the stationary points determined from geometry optimizations has been verified from vibrational analysis. This should be explicitly indicated.

The following sentence has been added

The nature of all the stationary points determined from geometry optimizations has been established on the basis of the harmonic frequency data from MP2/6$311++G(d, p)$ calculations.

The basis set used in CCSD(T) calculations should be indicated (page 6).

The revised sentence looks as following:

The conclusions obtained from the MP2 results were additionally verified by single point calculations at the coupled cluster method with single and double excitations, and its extension with the perturbative estimation of the contribution due to 3-body clusters at the CCSD(T)/aug-cc-pvdz//MP2/aug cc-pvdz level of theory.

It is not clear to me whether references 27 (after eq. 1) and 30 (together with ref. 26) are properly placed in the text (page 7).

We have found one misprinting and removed reference 30 from page 7.

There is a mistake in the equation for the equilibrium constant (paragraph 3).

The mistake has been corrected 
What is the meaning of the sign ' at the end of the paragraph 6 in page $8 ?$

The sign ' was removed.

Looking at the results in Table 1, the energy gap mentioned in the first paragraph of page 9 should be $0.49-1.17 \mathrm{kcal} / \mathrm{mol}($ ?). Even taking the original range $(0.64-1.02$ $\mathrm{kcal} / \mathrm{mol}$ ), I think that the authors are change the statement that "at least three 9Hspecies as virtually the same" (page 9). Let me simply notice the differences in population obtained for the different tautomers in Tables 2 and 3 . If the authors mean that the uncertainties due to computational methods are very large, this should be clearly stated

The range of the relative energies has been changed to $0.49-1.17 \mathrm{kcal} / \mathrm{mol}$.

The revised paragraph reads as follows:

The comparison of these data with the results using different techniques which extrapolate the results to the complete basis set slightly changed the picture. $7 \mathrm{H}$-oxoamino-tautomer continues to possesses the lowest energy. However, on the first glance the order of relative energies is not the constant and depends on the extrapolation methods used. The more careful look on the data presented in Table 1 enable us to make different conclusion. First of all we would like to highlight that all extrapolation methods suggest significantly lower energy gap between the lowest tautomer and the last one. This energy gap is predicted in the range of 0.49-1.17 kcal/mol. Such a small energy difference approaches to the accuracy of applied quantum chemical methods.

It seems that the values given in Tables 2 and 3 for $9 \mathrm{H}$-syn-hydroxoamino are wrong, at least for those corresponding to columns 6 and 7, which should be exchanged. This will also change the respective populations of the tautomers.

We have carefully checked our files and did not find any mistakes there.

A brief discussion about the effect of temperature on the population of tautomers should be made in page 9 (section 3.2 and Tables 2 and 3 ).

The following sentence has been added.

In particular, relatively small difference c.a. $0.1 \mathrm{kcal} / \mathrm{mol}$ between corresponding values of relative enthalpy and relative Gibbs free energy result in significant change of the composition of equilibrium mixture.

* The definition of $9 \mathrm{H}-\mathrm{oxo}$ as reagent in the conversion from $9 \mathrm{H}-\mathrm{oxo}$ to $9 \mathrm{H}$-hydroxo forms cannot be given from the preferential population of the former tautomer in 
"crystal and living cells" (page 10), which are clearly different from the gas phase environment. The correct justification would come from the fact the experimental assays were performed by using crystals, where the oxo form should predominate (as explained at the end of the second paragraph in page 11, and first line in page 4).

We have already changed the corresponding paragraph in our manuscript (see our modifications placed on the p. 3 of this letter.

* According to the first conclusion, there is a discrepancy between the calculated stabilities of tautomers and the experimental data (ref 10, where a claim is made to support the existence of the $\mathbf{7 H}$-hydroxo tautomer). In ref $\mathbf{1 0}$, this is interpreted in terms of the failure of the Bolztmann description for the population obtained in laser desorption experiments. Some comments should be made in the text.

The following paragraph has been added

Hence, one can conclude that most likely the tautomeric mixture observed experimentally is not equilibrated neither at $0 \mathrm{~K}$, nor at room temperature. In other words the experimental conditions might not correspond to the thermodynamic equilibrium, the results of calculations should not be compare to experimentally determined relative concentration of different guanine tautomers

The meaning of the different rate constants shown in Table 4 should be indicated as a footnote.

The footnote has been added to Table 4:

$k_{0}^{f}, k_{298}^{f}$ denote the rate constants for the forward reaction of tautomerization in the temperature $0 \mathrm{~K}$ and $298 \mathrm{~K}$, respectively.

$k_{0}^{r}, k_{298}^{r}$ denote the rate constants for the reverse reaction of tautomerization in the temperature $0 \mathrm{~K}$ and $298 \mathrm{~K}$, respectively.

Referee 66.

The authors suggested that the errors introduced during the synthesis of DNA can be further decreased by hydration and corrected by a "proofreading" mechanism sustained by DNA polymerases. It could be a further evidence of the following paper which described a protecting role of water on the proton transfer process. [J. Phys. Chem. B. 2004, 108, 12999-13007.]

The suggested paper is cited and the commented upon in the text as follows:

However, the influence of mentioned factors should also be investigated with care, since, for example, recent data suggest that influence selective amount of water molecules stabilize or destabilize canonic forms depending on the position of a hydration. ${ }^{39}$ 
There are some minor errors. Such as on P11, "significantly higher then the barrier" should be "significantly higher than the barrier".

The misprinting has been corrected

\section{Referee 67}

Abstract : the sentence about the barriers does not correspond to the results of Table 4 ( 2 types of barriers with different heights)

We have edited this sentence and it reads now as follows:

The transition states of the following reversible reactions:

9H-oxo-amino-guanine $\rightleftharpoons 9 H$-syn-hydroxo-amino-guanine $\rightleftharpoons 9 H$-anti-hydroxo-amino-guanine have been studied.

We put this labels at unfigure notes according to suggestion of Referee 65 .

Page 9; item 1. I would make the following remark :

One observes experimentally comparable abundances for 4 conformers. Assuming a thermodynamic equilibrium, like the authors do, one has to conclude that the four energies should be close to the other by less than something like $.3 \mathrm{kcal} / \mathrm{mol}$. This seems difficult to assume because it is unlikely that the energy of the $7 \mathrm{H}$ oxoamino may be wrong by $4 \mathrm{kcal} / \mathrm{mol}$.

Another alternative is that there is no equilibrium. This might be due to the fact that, in all the experiments reported so far, the species studied are laser-desorbed, starting from a guanine crystal, with nearly $100 \%$ of oxo amino form. After light absorption and a fast temperature increase to several hundreds of ${ }^{\circ} \mathrm{C}$, a fast expansion of the vaporized material takes place in the vacuum, followed by a drastic cooling, first in the desorption/ablation plume and then in the supersonic expansion. Because all these events take place within typically a few tens of $\mu \mathrm{s}$, the time evolution of the systems might be too fast for a thermal equilibrium to be reached. In such a case the final tautomer distribution does not necessarily reflect the energetic order.

In my opinion, this might be the origin for the disagreement observed between experiment and theory. I think this should be expressed more clearly in the paper.

We have revised the corresponding paragraph of our paper which reads now as follows: 
mixture should be dominated by four tautomers which correspond to the lowest-energy forms of guanine found in this study. However, only three of them are those of the four tautomers observed experimentally. According to our data the equilibrium concentration of $7 \mathrm{H}$-syn-hydroxo-amino-guanine, which possibly was observed in experiments of Mons et al. ${ }^{10}$, should be negligible. There seems to be hardly a chance that improving upon the basis sets and computational methods would lead to large enough lowering of its relative energy and make its equilibrium concentration observable. Hence, one can conclude that most likely the tautomeric mixture observed experimentally is not equilibrated neither at $0 \mathrm{~K}$, nor at room temperature. In other words the experimental conditions might not correspond to the thermodynamic equilibrium, the results of calculations should not be compare to experimentally determined relative concentration of different guanine tautomers

In addition we would like to mention that the statement that the experimentally observed mixture is not equilibrated was placed in the conclusion \#2 of the original version of the manuscript and we kept it in the revised version. 